\title{
A Retrospective Observational Analysis of Clinical Outcomes before and after the Publication of the AACE/ACE Guidelines
}

\author{
Rajesh Peddaiahgari' ${ }^{1}$, Ayoade 0. Adeyemi ${ }^{1}$, Jamie C. Barner ${ }^{1}$, Debra A. Lopez ${ }^{1}$, \\ Jason R. Jokerst ${ }^{2}$ \\ ${ }^{1}$ The University of Texas, College of Pharmacy, Austin, USA \\ ${ }^{2}$ CommUnityCare Clinics, Austin, USA \\ Email: ayoade adeyemi@yahoo.ca
}

Received 15 April 2014; revised 14 May 2014; accepted 13 June 2014

Copyright (C) 2014 by authors and Scientific Research Publishing Inc.

This work is licensed under the Creative Commons Attribution International License (CC BY). http://creativecommons.org/licenses/by/4.0/

(c) () Open Access

\section{Abstract}

Background: The influence of the American Association of Clinical Endocrinologists/American College of Endocrinology (AACE/ACE) guidelines (hereafter, "guidelines") on clinical outcomes of patients with diabetes is yet to be assessed. Objectives: To determine if differences occurred in type of: 1) medication class prescribed; 2) therapy by A1c strata in type 2 diabetic (T2DM) patients before and after guidelines were published (December 2009). Methods: Data for this retrospective cohort study were extracted from community health center clinics' electronic medical records for patients who: 1) were adults (18 - 80 years) with T2DM; and 2) had at least one A1c value before and after guidelines. Demographic characteristics and clinical outcomes [oral antidiabetic (OAD) medication class, therapy type (mono, dual, triple), and A1c values] were collected. A1c was stratified into four levels: $<6.5 ; 6.5$ - 7.5; 7.6 - 9.0; >9.0. Descriptive and inferential statistics were used. Results: The random sample of 302 patients was $55.4 \pm 11.7$ years of age, primarily female $(65.9 \%)$ and Hispanic (68.8\%). Regarding medication class, most (68.5\% before and $72.2 \%$ after guidelines) patients were prescribed metformin. The proportion of patients across individual medication classes increased significantly $(p<0.05)$ between the periods before and after guidelines, with the greatest percentage increase observed with dipeptidyl peptidase-4 (DPP-4) inhibitors $(36.2 \%)$. Chi-square results revealed a significant difference $(p<0.0001)$ between type of therapy and A1c strata. Before guidelines, $55.7 \%$ of patients with A1c values $<6.5 \%$ were on monotherapy, while $44.1 \%$ of patients with A1c values $>9 \%$ were on dual therapy. After guidelines, $48.4 \%$ of patients with A1c values $<6.5 \%$ were on monotherapy, while $31.8 \%$ of patients with A1c values $>9 \%$ were on dual therapy. Almost one-half $(48.3 \%)$ of patients remained in the same A1c strata before and after guidelines were published and there were no significant changes in mean A1c. Conclusions: DPP-4 inhibitor use showed the largest increase after guidelines were issued, however, there were no improvements in A1c. Additional research is warranted to eva- 
luate healthcare providers' adherence to AACE/ACE guidelines and how this influences patients' health outcomes.

Keywords

AACE/ACE Guidelines, Type 2 Diabetes, Non-Insulin Antidiabetic Medications, HbA1c

\section{Introduction}

Diabetes is currently the seventh leading cause of death in the US with over 25 million people presenting with the disease. In 2007, diabetes (both type 1 and 2) accounted for over \$174 billion in healthcare costs with over $90 \%$ of this cost attributed to the management of type 2 diabetes (T2DM) alone [1] [2]. Adequate management of T2DM is crucial to improving affected patients' health related quality of life and productivity. However, due to the chronic and complex nature of the disease and its associated complications, management remains challenging [3]. In an attempt to assist healthcare providers in improving diabetes disease management, several treatment guidelines and consensus statements have been released [4]-[6]. These guidelines include the American Diabetes Association (ADA), Veterans Health Administration/US Department of Defense (VA/DOD) and the International Diabetes Federation (IDF) guidelines [5] [7] [8]. These guidelines are focused on improving clinical outcomes and preventing or minimizing associated risks of complications by providing standards of care that focus on achieving and sustaining optimal glycemic control [5]. The FDA's approval of new antidiabetic medications in recent years, coupled with results from a number of recent clinical trials made it pertinent to reexamine recommended therapies from previous algorithms [6].

In December 2009, the American Association of Clinical Endocrinologists/American College of Endocrinology (ACCE/ACE) published treatment guidelines with two distinctive additions to similar guidelines. These additions include stratification of treatment algorithms based on patients' HbA1c (A1c) levels, as well as, a recommendation to include dipeptidyl peptidase 4-inhibitors (DPP-4 inhibitors) or glucagon-like peptide 1-agonists (GLP-1 agonists) as add-on therapy when monotherapy is not sufficient [6]. The recommendation for the inclusion of either DPP-4 inhibitors or GLP-1 agonists to therapy is due to decreased hypoglycemia when compared to other insulin secretagogues. Furthermore, the addition of thiazolidinediones, glinides or sulfonylureas has been recommended where triple therapy is required [6].

While treatment guidelines have been established through clinical trials to achieve tight glycemic control in T2DM [9], it is however disappointing that treatment outcomes have not improved in some clinical practices [10] [11]. Although the influence of the recently published AACE/ACE guidelines on health outcomes has not yet been assessed, some studies have suggested that physicians' adherence to a number of guidelines is sub-optimal [12]-[15]. It is expected that the availability of studies that show how physicians' adherence to these guidelines influences health outcomes (such as achieving the desired glycemic control while minimizing the risk for microvascular and macrovascular complications) with real life data would encourage adherence to these guidelines and hence improve outcomes. This study aims to assess the influence of the publication of the ACCE/ACE guidelines on health outcomes in diabetic patients on non-insulin antidiabetic medications.

\section{Materials and Methods}

\subsection{Data Source}

Data were obtained from CommUnityCare clinics, which are federally qualified health centers and joint commission accredited clinics that provide healthcare services to the underserved population in the Travis County area of Texas. This study was approved by the Institutional Review Board (IRB) Committee of The University of Texas at Austin and all study protocols were met.

\subsection{Study Population}

A random sample of patients was drawn from a retrospective cohort of patients with T2DM. Patients included in this study were between 18 and 80 years of age with a diagnosis of T2DM and with at least one A1c value with- 
in 12 months before and after the index period. The index period was defined as 3 months after the guidelines' publication date in December 2009 (i.e., December 31, 2009-March 31, 2010). A 3-month window after the guidelines were published was used to provide a grace period for the following reasons: 1) A1c is a 3-month average of blood glucose levels and 2) to allow dissemination and adoption of the new guidelines. Eligible patients who received care from CommUnityCare clinics and who were on non-insulin antidiabetic medication therapy were followed from January 2009 to December 2010, that is, 12-months before and 12 months after the guidelines.

\subsection{Study Variables}

\subsubsection{Dependent Variables}

The main dependent variable was change in A1c levels before and after guidelines. Before the guidelines were issued, A1c value was defined as the initial A1c value prior to, but closest to index date. After the guidelines, A1c value was defined as any A1c value obtained 3-months after the guidelines were released. The other dependent variables include: 1) change in non-insulin antidiabetic medications (i.e., metformin, sulfonylureas, thiazolidinediones, DPP-4 inhibitors and GLP-1 agonists) used; 2) change in type of therapy (e.g., monotherapy, dual therapy, etc); and 3) change in A1c strata (i.e., <6.5\%, 6.5\% - 7.5\%, 7.6\% - 9.0\% and $>9.0 \%$ ) before and after the guidelines. Only non-insulin antidiabetic medications were included in the type of therapy combinations.

\subsubsection{Independent Variables}

The publication of the new AACE/ACE guidelines in December 2009 was the main independent variable. Covariates included in the study include age, gender, and race.

\subsection{Data Collection and Analysis}

This was a retrospective cohort study involving the extraction of data from the CommUnityCare electronic medical records. Descriptive statistics (i.e., mean, standard deviation (SD), percentages and frequencies) were used to describe the study population while chi-square statistics were used to assess changes in clinical outcomes before and after guidelines. An a priori significance level of $\mathrm{p}<0.05$ was assumed and all statistical analyses were carried out using SAS 9.2 version (SAS Institute Inc. Cary, North Carolina).

\section{Results}

A random sample of 302 patients was drawn from those who met the inclusion criteria $(\mathrm{N}=2865)$. The average age was $55.4 \pm 11.7$ years, majority were female $(\mathrm{N}=199 ; 65.9 \%)$ and Hispanic $(\mathrm{N}=208 ; 68.8 \%)$. Table 1 shows the chi-square results of medication class utilization before and after the guidelines' publication. Overall, medication utilization increased with the exception of GLP-1 agonists. GLP-1 agonists were not prescribed by providers to treat T2DM in this patient population. Percentage increase in medication utilization ranged from 5.4\% (metformin) to 36.2\% (DPP-4 inhibitors). The patients with the highest frequency of change were those who had DDP-4 inhibitors added to their therapy regimen (35.9\%). The type of therapy prescribed was also assessed to determine if there were changes before and after guidelines.

Table 1. Chi-square analysis of medication class changes before and after the publication of AACE/ACE guidelines ${ }^{\mathrm{a}}$.

\begin{tabular}{|c|c|c|c|}
\hline Medication Class & Before N (\%) & After N (\%) & $\%$ Change (before vs after) \\
\hline Metformin & $207(68.5)$ & $218(72.2)$ & *5.4\% increase \\
\hline Sulfonylureas (glipizide, glimepiride, glyburide) & $121(40.1)$ & $133(44.0)$ & *9.7\% increase \\
\hline Thiazolidinediones (pioglitazone, rosiglitazone) & $56(18.5)$ & $60(19.8)$ & ${ }^{*} 7.0 \%$ increase \\
\hline DPP-4 inhibitors (sitagliptin, saxagliptin, linagliptin) & $25(8.3)$ & $34(11.3)$ & ${ }^{*} 36.2 \%$ increase \\
\hline GLP-1 agonists (exenatide, liraglutide) & $0(0.0)$ & $0(0.0)$ & No change \\
\hline
\end{tabular}

${ }^{\mathrm{a}}$ Totals will add to more than $100 \%$ due to multiple therapies; ${ }^{*}$ Statistically significant at $\mathrm{p}<0.05$. 
Table 2 shows that before and after the guidelines, the plurality of patients was on mono or dual therapy. That is, before the publication of the guidelines, $38.1 \%$ and $30.5 \%$ of patients were on monotherapy and dual therapy, respectively, while $35.8 \%$ and $31.1 \%$ were on monotherapy and dual therapy after the guidelines' publication, respectively. There was also a slight decrease in the proportion of patients who had no medication before (19.5\%) and after $(17.2 \%)$ the guidelines and an increase in the proportion of patients on triple therapy or more before (11.9\%) and after (15.9\%) the guidelines were published. Mean A1c values before and after guidelines were 8.0\% ( \pm 2.0$)$ and $8.2 \%( \pm 2.1)$, respectively, and were not significantly different.

Chi-square analysis revealed that before the guidelines' publication, the type of therapy differed significantly ( $<<0.0001$ ) by A1c strata (see Table 3). Of patients in the $<6.5 \%$ A1c strata, the majority $(55.7 \%)$ were on monotherapy. For the $6.5 \%-7.5 \%$ and the $7.6 \%-9.0 \%$ A1c strata, patients were either on mono (35.5\% vs $36.5 \%$, respectively) or dual therapy (31.1\% vs $35.1 \%$, respectively), while the plurality (44.1\%) of patients in the $>9.0 \%$ A1c stratum were on dual therapy. Overall, the results showed a slight increase in the number of medications as A1c levels increased.

Similar trends were observed after the guidelines were published (Table 4).

Further chi-square analyses (see Table 5$)$ revealed a significant $(\mathrm{p}<0.0001)$ relationship in A1c strata before

Table 2. Chi-square analysis of changes in therapy type before and after publication of AACE/ACE guidelines.

\begin{tabular}{ccc}
\hline Therapy Type & Before N (\%) & After N (\%) \\
\hline No medication & $59(19.5)$ & $52(17.2)$ \\
Monotherapy & $115(38.1)$ & $108(35.8)$ \\
Dual therapy & $92(30.5)$ & $94(31.1)$ \\
$\geq 3$ medications & $36(11.9)$ & $48(15.9)$ \\
Total & $\mathbf{3 0 2}(\mathbf{1 0 0 . 0 )}$ & $\mathbf{3 0 2}(\mathbf{1 0 0 . 0})$ \\
\hline
\end{tabular}

$\mathrm{X}^{2}=467.24 ; \mathrm{p}<0.0001$

Table 3. Type of therapy by A1c strata before the AACE/ACE guidelines $(\mathrm{N}=302)$.

\begin{tabular}{|c|c|c|c|c|}
\hline \multirow[b]{2}{*}{ Therapy Type } & \multicolumn{4}{|c|}{ A1c Strata } \\
\hline & $<6.5 \mathrm{~N}(\%)$ & 6.5 - 7.5 N (\%) & $7.6-9.0 \mathrm{~N}(\%)$ & $>9.0 \mathrm{~N}(\%)$ \\
\hline No therapy & $22(31.4)$ & 15 (16.7) & $10(13.5)$ & $12(17.7)$ \\
\hline Monotherapy & 39 (55.7) & $32(35.5)$ & 27 (36.5) & $17(25.0)$ \\
\hline Dual therapy & $8(11.4)$ & $28(31.1)$ & $26(35.1)$ & $30(44.1)$ \\
\hline Triple therapy or more & $1(1.5)$ & 15 (16.7) & $11(14.9)$ & $9(13.2)$ \\
\hline Total & $70(100.0)$ & $90(100.0)$ & $74(100.0)$ & $68(100.0)$ \\
\hline
\end{tabular}

$\mathrm{X}^{2}=37.8 ; \mathrm{p}<0.0001$

Table 4. Type of therapy by A1c strata after the AACE/ACE guidelines $(\mathrm{N}=302)$.

\begin{tabular}{|c|c|c|c|c|}
\hline \multirow[b]{2}{*}{ Therapy Type } & \multicolumn{4}{|c|}{ A1c Strata } \\
\hline & $<6.5$ N (\%) & $6.5-7.5 \mathrm{~N}(\%)$ & 7.6 - $9.0 \mathrm{~N}(\%)$ & $>9.0 \mathrm{~N}(\%)$ \\
\hline No therapy & $17(27.4)$ & $12(14.1)$ & $6(8.6)$ & $17(20.0)$ \\
\hline Monotherapy & $30(48.4)$ & 33 (38.8) & $22(31.4)$ & $23(27.0)$ \\
\hline Dual therapy & 9 (14.5) & $30(35.3)$ & $28(40.0)$ & 27 (31.8) \\
\hline Triple therapy or more & $6(9.7)$ & $10(11.8)$ & $14(20.0)$ & $18(21.2)$ \\
\hline Total & $62(100.0)$ & $85(100.0)$ & $70(100.0)$ & $85(100.0)$ \\
\hline
\end{tabular}

$\mathrm{X}^{2}=25.2 ; \mathrm{p}=0.0028$ 
Table 5. Comparison of A1c by strata before and after guidelines $(\mathrm{N}=302)$.

\begin{tabular}{|c|c|c|c|c|c|}
\hline \multicolumn{6}{|c|}{ A1c Strata \% } \\
\hline \multicolumn{2}{|c|}{ After } & $<6.5$ N (\%) & $6.5-7.5 \mathrm{~N}(\%)$ & $7.6-9.0 \mathrm{~N}(\%)$ & $>9.0 \mathrm{~N}(\%)$ \\
\hline \multirow{4}{*}{ Before } & $<6.5$ & $39(12.9)^{1}$ & $20(6.6)^{3}$ & $4(1.3)^{3}$ & $7(2.2)^{3}$ \\
\hline & $6.5-7.5$ & $15(5.0)^{2}$ & $37(12.3)^{1}$ & $23(7.6)^{3}$ & $15(5.0)^{3}$ \\
\hline & $7.6-9.0$ & $6(2.0)^{2}$ & $21(7.0)^{2}$ & $27(8.9)^{1}$ & $20(6.6)^{3}$ \\
\hline & $>9.0 \%$ & $2(0.7)^{2}$ & $7(2.2)^{2}$ & $16(5.3)^{2}$ & $43(14.2)^{1}$ \\
\hline
\end{tabular}

$\mathrm{X}^{2}=129.0 ; \mathrm{p}<0.0001 .{ }^{1}$ Same stratum maintained before vs after the AACE/ACE guidelines publication $(\mathrm{N}=146) ;{ }^{2}$ Lower stratum observed before vs after the AACE/ACE guidelines publication $(\mathrm{N}=67) ;{ }^{3}$ Higher stratum observed before vs after the AACE/ACE guidelines publication $(\mathrm{N}=89)$.

and after the guidelines' publication. When A1c strata were compared before and after the guidelines, a majority of patients, $48.3 \%(\mathrm{~N}=146)$, remained within their initial respective A1c stratum. Among patients who changed strata, 22.2\% $(\mathrm{N}=67)$ of patients had improved A1c values and 29.5\% $(\mathrm{N}=89)$ of patients had worsened A1c values after the guidelines.

\section{Discussion}

The primary objective of this study was to determine if there was a significant difference in the treatment of diabetes after the publication of the AACE/ACE guidelines by evaluating the type of medication, type of therapy prescribed for treatment, and A1c reduction. Based on medication utilization, it was observed that the use of all classes of non-insulin antidiabetic medications with the exception of GLP-1 agonists increased after the AACE/ ACE guidelines were released, though exenatide and liraglutide (GLP-1 agonists) were approved in April 2005 and January 2010, respectively. High cost, possible gastrointestinal disturbances and the dosage form (injections) may be reasons why there was no utilization in this practice setting [16]. Additionally, during the time of this study, the use of GLP-1 agonist was not approved for concomitant use with insulin. This was not the case with DDP-4 inhibitors, which were approved for concomitant use with insulin. Another possible reason why liraglutide may not have been used may be that although the AACE/ACE guidelines recommends GLP-1 agonists as second OAD medication of choice after metformin in patients with elevated fasting and post-prandial glucose levels; liraglutide was not specifically mentioned in the guidelines [4] [6]. After the guidelines, DPP-4 inhibitor use increased by $36.2 \%$, which was higher than the percentage of patients who had a sulfonylurea added to their therapy (9.7\%). This change is congruent with the AACE/ACE guideline recommendation of using DPP-4 inhibitors earlier in therapy and reducing the use of sulfonylurea agents to minimize hypoglycemic events [6]. Depending on patients' A1c values, the AACE/ACE guidelines recommend using a combination of medications to treat diabetes. The use of more than 1 medication is usually associated with higher A1c levels, an indication of inadequately controlled diabetes. The observed increase in the proportion of patients on dual and at least triple therapy before and after the guidelines suggests that patients with poorly controlled diabetes were placed on regimens that were better suited to control their A1c levels. Although a slight increase in mean A1c was observed before and after guidelines, t-test results showed that this difference was not significant, however, chi-square analyses revealed a significant relationship between A1c strata between these two periods. Generally, almost one-half of the patients neither improved nor deteriorated over the study period based on their A1c values, the gradual progression of the disease and possibly poor adherence to medications and lifestyle modifications may be likely reasons for the insignificant change in A1c values observed in this group of patients. Some changes were observed to be congruent with the AACE/ACE guidelines; thus, suggesting that the AACE/ACE guidelines had some impact on prescribing patterns in this practice setting. However, in view of the severe implications of inadequate management of this chronic disease, greater improvements in A1c levels would imply better provider adherence to the guidelines and improved management of diabetes, as well as patient adherence to recommended therapies. More studies are needed to evaluate how the AACE/ACE guidelines influence the management of diabetes and patient health outcomes, especially now that adequate time has elapsed since the dissemination of the guidelines. 


\section{Limitations}

Insulin was not considered as add-on antidiabetic therapy with other medications, which could affect results, especially for patients with high A1c values. Dose and frequency of the antidiabetic medications were not evaluated. This could affect the results since T2DM can be treated with an increase in the dose and/or frequency of the medication. Adequate time may be required after the dissemination of the guidelines before significant effects of their acceptance and adherence by healthcare providers can be evaluated. Since the publication of the guidelines was used as proxy for its use, it cannot be ascertained whether the observed outcomes were attributed to healthcare physicians' adherence to the guidelines.

\section{Conclusion}

The changes in prescribing patterns that were observed, notably increase in DPP-4 inhibitors, may be associated with healthcare providers' adoption of the AACE/ACE guidelines. However, more studies are required to support the observed findings in other settings and populations. It is also necessary to evaluate how health outcomes (in terms of A1c values) are influenced by the adoption of these guidelines.

\section{References}

[1] Centers for Disease Control and Prevention (2011) National Diabetes Fact Sheet: National Estimates and General Information on Diabetes and Prediabetes in the United States. Centers for Disease Control and Prevention, Atlanta.

[2] Centers for Disease Control and Prevention (2010) Diabetes: Successes and Opportunities for Population-Based Prevention and Control. http://www.cdc.gov/nccdphp/publications/aag/pdf/diabetes.pdf

[3] Brown, J.B., Harris, S.B., Webster-Bogaert, S., Wetmore, S., Faulds, C. and Stewart, M. (2002) The Role of Patient, Physician and Systemic Factors in the Management of Type 2 Diabetes Mellitus. Family Practice, 19, 344-349. http://dx.doi.org/10.1093/fampra/19.4.344

[4] Nathan, D.M., Buse, J.B., Davidson, M.B., et al. (2009) Medical Management of Hyperglycemia in Type 2 Diabetes: A Consensus Algorithm for the Initiation and Adjustment of Therapy: A Consensus Statement of the American Diabetes Association and the European Association for the Study of Diabetes. Diabetes Care, 32, 193-203. http://dx.doi.org/10.2337/dc08-9025

[5] American Diabetes Association (2009) Standards of Medical Care in Diabetes_-2009. Diabetes Care, 32, S13-S61. http://dx.doi.org/10.2337/dc09-S013

[6] Rodbard, H.W., Jellinger, P.S., Davidson, J.A., et al. (2009) Statement by an American Association of Clinical Endocrinologists/American College of Endocrinology Consensus Panel on Type 2 Diabetes Mellitus: An Algorithm for Glycemic Control. Endocrine Practice, 15, 540-559. http://dx.doi.org/10.4158/EP.15.6.540

[7] The Management of Diabetes Mellitus Working Group (1999) VHA/DOD Clinical Practice Guideline for the Management of Diabetes in the Primary Care Setting. http://www.va.gov/diabetes/docs/Clinical_Practice_Guidelines.doc

[8] International Diabetes Federation CGT (2005) Global Guideline for Type 2 Diabetes. http://www.idf.org/webdata/docs/IDF\%20GGT2D.pdf

[9] Liday, C. (2011) Overview of the Guidelines and Evidence for the Pharmacologic Management of Type 2 Diabetes Mellitus. Pharmacotherapy, 31, 37S-43S. http://dx.doi.org/10.1592/phco.31.12.37S

[10] Levetan, C. (2007) Oral Antidiabetic Agents in Type 2 Diabetes. Current Medical Research and Opinion, 23, $945-952$. http://dx.doi.org/10.1185/030079907X178766

[11] Cohen, A. and Horton, E.S. (2007) Progress in the Treatment of Type 2 Diabetes: New Pharmacologic Approaches to Improve Glycemic Control. Current Medical Research and Opinion, 23, 905-917. http://dx.doi.org/10.1185/030079907X182068

[12] Larme, A.C. and Pugh, J.A. (2001) Evidence-Based Guidelines Meet the Real World: The Case of Diabetes Care. Diabetes Care, 24, 1728-1733. http://dx.doi.org/10.2337/diacare.24.10.1728

[13] Grant, R.W., Wexler, D.J., Watson, A.J., et al. (2007) How Doctors Choose Medications to Treat Type 2 Diabetes: A National Survey of Specialists and Academic Generalists. Diabetes Care, 30, 1448-1453. http://dx.doi.org/10.2337/dc06-2499

[14] Francke, A.L., Smit, M.C., de Veer, A.J. and Mistiaen, P. (2008) Factors Influencing the Implementation of Clinical Guidelines for Health Care Professionals: A Systematic Meta-Review. BMC Medical Informatics and Decision Making, 8, 38. http://dx.doi.org/10.1186/1472-6947-8-38

[15] Cabana, M.D., Rand, C.S., Powe, N.R., et al. (1999) Why Don’t Physicians Follow Clinical Practice Guidelines? A 
Framework for Improvement. Journal of the American Medical Association, 282, 1458-1465. http://dx.doi.org/10.1001/jama.282.15.1458

[16] Derosa, G. and Maffioli, P. (2012) Glp-1 Agonists Exenatide and Liraglutide: A Review about their Safety and Efficacy. Current Clinical Pharmacology, 7, 214-228. 
Scientific Research Publishing (SCIRP) is one of the largest Open Access journal publishers. It is currently publishing more than 200 open access, online, peer-reviewed journals covering a wide range of academic disciplines. SCIRP serves the worldwide academic communities and contributes to the progress and application of science with its publication.

Other selected journals from SCIRP are listed as below. Submit your manuscript to us via either submit@scirp.org or Online Submission Portal.
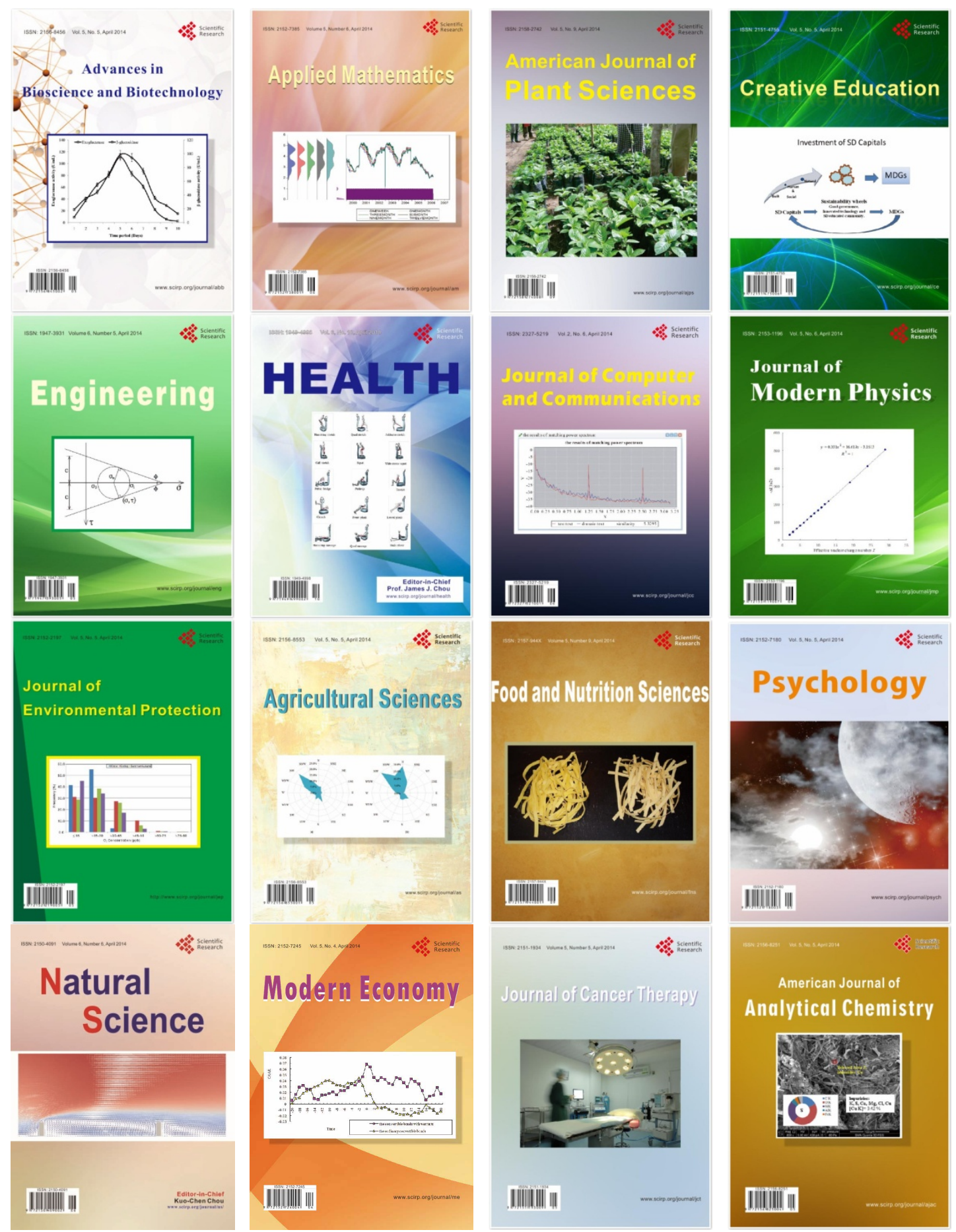